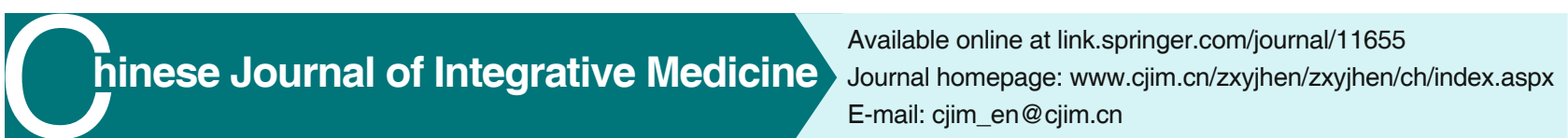

Original Article

\title{
Effect of Jiuwei Zhuhuang Powder on Cough Resolution in Children with Upper Respiratory Tract Infections: A Multicenter Randomized Controlled Trial*
}

\author{
LUO Hui ${ }^{1}$, SONG Gui-Hua ${ }^{2}$, MA Xiao-jian ${ }^{3}$, SUN Meng-meng ${ }^{2}$, ZHANG Man ${ }^{4}$, \\ XIE Jian-rong ${ }^{5}$, and PENG Shao ${ }^{*}$
}

\begin{abstract}
Objective: To assess the effectiveness of Jiuwei Zhuhuang Powder (JWZH), a Tibetan patent medicine in treating upper respiratory tract infection (URTI) associated cough in children. Methods: The study was a multicenter, randomized, open-label, controlled trial. A total of 142 children aged 2 to 14 years old, with URTIassociated cough within $48 \mathrm{~h}$ of onset, were randomly assigned to two groups at a 1:1 ratio by computer-generated randomization sequence. Children were treated with JWZH (1 to $1.5 \mathrm{~g}$, twice to thrice daily) in the treatment group or conventional treatment (Pediatric Paracetamol, Artificial Cow-bezoar and Chlorphenamine Maleate Granules, 0.25 to $1 \mathrm{~g}$, thrice daily) in the control group for 5 days. The primary endpoints were the time to cough resolution and 4-day cough resolution rate. The secondary endpoints were the daily improvement in symptom scores and cough resolution rate during the study period. Results: A total of 138 children were included in the intention-to-treat analysis, with 71 cases in the treatment group and 67 cases in the control group. Compared with the conventional treatment, the children receiving JWZH had a shorter time to cough resolution [hazard ratio, 2.10; $95 \%$ confidence interval $(\mathrm{Cl}), 1.29-3.40 ; P=0.003]$. The median time to cough resolution for children receiving JWZH was shorter than that of the conventional treatment ( 2 days vs. 3 days; $P<0.001$ ). The 4-day cough resolution rate in the JWZH group was higher than that of the control group (94.4\% vs. $74.6 \%$; risk difference: $19.8 \%, 95 \% \mathrm{Cl}: 8.1 \%-31.5 \%$; relative risk: $1.265,95 \% \mathrm{Cl}: 1.088-1.470 ; P=0.001)$. There were no statistically significant differences in the improvement of other symptoms caused by URTI $(P>0.05)$. Adverse events was reported in $5.6 \%(4 / 71)$ and $4.5 \%(3 / 67)$ in participants of JWZH and PPACCM groups $(P>0.05)$, respectively, which were all mild and resolved without treatment. Conclusion: JWZH seemed to be a safe and effective therapy for URTI-associated cough in children. (Trial registration No. ChiCTR2000039421)

KEYWORDS child cough, upper respiratory tract infection, Jiuwei Zhuhuang Powder, randomized controlled trial, Tibetan medicine
\end{abstract}

Cough is among the most common symptoms associated with upper respiratory tract infections (URTI), and children are more likely to experience URTI-associated acute cough than adults. ${ }^{(1)}$ Acute cough is present in approximately $70 \%$ of URTI at onset or day one, and persists for the first week in more than $50 \%$ of children. ${ }^{(2,3)}$ URTI-associated cough causes health, social and economic problems with significant implications for children, parents and the health care system. ${ }^{(4)}$ Children with URTI-associated cough may have symptoms of discomfort, absent from daycare or school, and at risk of developing complications and receiving overprescribed antibiotic treatment. ${ }^{(5)}$ Parents may miss workdays and spend billions of dollars on worthless remedies when their children have a URTI and cough episode. ${ }^{(4)}$ Since generally caused by viruses, the management of URTI is symptomatic, and antibiotics are not effective in children or adults. The common treatments for cough

CThe Chinese Journal of Integrated Traditional and Western Medicine Press and Springer-Verlag GmbH Germany, part of Springer Nature 2021

*Supported by the National Natural Science Foundation of China (No. 81704197)

1. Institute for Tibetan Medicine, China Tibetology Research Center, Beijing (100101), China; 2. Department of Pediatrics, the First Affiliated Hospital of Henan University of Chinese Medicine, Zhengzhou (450000), China; 3. Department of Pediatrics, Huaihe Hospital of Henan University, Zhengzhou (450000), China; 4. Department of Pediatrics, the First Affiliated Hospital of Zhengzhou University, Zhengzhou (475000), China; 5. School of Statistics, Renmin University of China, Beijing (100872), China Correspondence to: Prof. PENG Shao, E-mail: Pengshaodh630@ 126.com

DOI: https://doi.org/10.1007/s11655-021-3462-x 
relief are antitussives, such as dextromethorphan. ${ }^{(6)}$ However, there is a potential for harm and no benefits for relieving children cough with over-the-counter cough and cold medications. ${ }^{(4,7-9)}$ Given the challenges of URTI-associated cough treatment, the search for alternative safe and effective approaches remains an area of active investigation.

Tibetan medicine constitutes an important part of medical system in China. ${ }^{(10)}$ Jiuwei Zhuhuang (JWZH), a Tibetan medicine formula, has been used to treat acute cough in children for hundreds of years. ${ }^{(11,12)}$ In 2002, JWZH Powder (九味筑黄散) was approved by the China Food and Drug Administration as a Tibetan patent medicine for the treatment of cough associated with upper respiratory tract viral infection and pneumonia in children. However, except for history materials, only two case series studies are available in the literature indicating that JWZH could relieve URTIassociated cough in children. ${ }^{(11,12)}$ There is lack of randomized controlled trial (RCT) evaluating the effects of JWZH in this condition. The aim of the study was to investigate whether the JWZH Powder could decrease the time to cough resolution and improve the 4-day cough resolution rate in children with URTI-associated cough compared with conventional treatment.

\section{METHODS}

\section{Study Design and Participants}

The study was a multicenter, randomized, openlabel, controlled trial conducted in three medical centers in China, including the First Affiliated Hospital of Zhengzhou University, the First Affiliated Hospital of Henan University of Traditional Chinese Medicine, and Huaihe Hospital of Henan University. The protocol was registered at chictr.org.cn (No. ChiCTR2000039421), and recruitment occurred from December 2019 through March 2021. Institutional review boards of the three hospitals reviewed and approved the protocol (SS-2019-070). Participants were recruited from the pediatric outpatient at the three centers. The parents of all participants signed written informed consent forms before enrollment.

Children aged 2 to 14 years old, regardless of gender, with URTI within $48 \mathrm{~h}$ of onset and with cough as the main complaint (cough score $\geqslant 2$ ) ${ }^{(13)}$ were eligible. URTI was diagnosed according to the China's guideline on standardized diagnosis and treatment of common cold in Children. ${ }^{(14)}$ Children were excluded if: (1) with severe malnutrition or other serious systemic diseases such as cardiovascular, liver, kidney and hematopoietic system; (2) had a history of allergy to the study drugs; (3) had taken antitussives or anti-cold medicine; or (4) had any conditions that prevented participation in the study, such as the condition has progressed to lower respiratory tract infection.

\section{Randomization and Procedure}

The eligible participants were randomly assigned to either the JWZH or control group. The allocation ratio was $1: 1$ ratio. Computer-generated random numbers were generated by a statistician not involved in the study. The group assignments were enclosed in opaque, sealed, consecutively numbered envelopes. The envelopes were opened only when an eligible participant was identified and the informed consent was provided, and the participant was placed on the allocated therapy accordingly.

Parents of the study children completed a form that included demographic items such as the child's age, sex, height and weight. Parents were also asked about the past medical history and exposure factors that potentially related to the duration and severity of URTI-associated cough, including the number of colds their child had experienced during the past 12 months, whether their child had asthma, variant cough, or hospitalization history due to respiratory diseases, and presence of cigarette smokers in the household. Subjective measurement of cough and other URTIassociated symptoms were recorded by the parents before treatment and at 1-5 days after treatment. During the study, the research assistants blinded to treatment assignments were responsible for the contact of the participants. They used a predetermined unified questioning method to contact the parents of children enrolled in this study via phone or WeChat (a Chinese multi-purpose messaging, social media and mobile payment application) once per day. The assistants' questions were limited to the outcome and whether to stop the medication, and did not involve the grouping, drug types and the remaining drug amount. At each contact, parents were required to provide the recorded patient diary. Symptoms, any clinical events, and the dose of the study drug were recorded. If a child recovered within 5 days, the contact would end $24 \mathrm{~h}$ after the symptoms disappear.

\section{Interventions}

JWZH Powder (Tibet Nyingchi Yutuo Tibetan 
Medicine Co. Ltd., Lhasa, China; batch No. 191004) was used in the treatment group. It consisted of 9 herbs: Bambusae textilis McClure, Crocus sativus, Bovis calculus artifactus, Bergeniae rhizome, Aconitum tanguticum, Glycyrrhizae radix et rhizome, Radix solms-laubachiae, Lagotis brevituba maxim, and Santali albi lignum. The dosages of each single component are shown in Appendix 1. Children aged 2 to 3 years old took $2 \mathrm{~g} / \mathrm{d}$ ( $1 \mathrm{~g}$, twice daily), 4 to 7 years old took $3 \mathrm{~g} / \mathrm{d}$ ( $1 \mathrm{~g}$, thrice daily), and 8 years old and above took $4.5 \mathrm{~g} / \mathrm{d}$ (1.5 g, thrice daily).

Participants in the control group received conventional treatment of Western medicine, using Pediatric Paracetamol, Artificial Cow-bezoar and Chlorphenamine Maleate Granules [PPACCM, Sunflower Pharmaceutical Group (Chongqing) Co. Ltd., China], a commonly used medication recommended by the guideline for treating children URTI in China. ${ }^{(14)}$ The dosages were based on the recommendations of manufacturers (0.25 to $1 \mathrm{~g}$, thrice daily). The study medication began after enrollment and continued until all symptoms had resolved, up to a maximum of 5 days. During the course of treatment, if the axillary temperature exceeded $38.0^{\circ} \mathrm{C}$, ibuprofen can be added orally under the guidance of the study pediatrician.

\section{Outcomes}

The primary outcomes were time to cough resolution and 4-day cough resolution rate. Cough resolution was defined as the cough disappeared continuously for $\geqslant 24 \mathrm{~h}$. Time to cough resolution was calculated from the randomization to the last cough occurred, and it was measured in half-days. The 4-day cough resolution rate was the proportion of participants with cough resolution on the fourth day of treatment.

The secondary outcomes included the daily improvement in symptom scores and cough resolution rate during the study period. The score of URTI symptoms included fever, daytime cough, nighttime cough, nasal congestion, runny nose, and sneeze ranged from 0 to 3 (0 absent, 1 mild, 2 moderate, and 3 severe). The scoring criteria adopted "The cough diagnosis and treatment guidelines" formulated by the Respiratory Branch of the Chinese Medical Association and "The design and evaluation technology of new Chinese medicines for acute URTI in children" formulated by the Pediatric Branch of the China
Association of Chinese Medicine. ${ }^{(13,15)}$ Symptom scores were recorded and compared with baseline scores until 5 days after treatment in all groups. The detailed scoring criteria are shown in Appendix 2.

\section{Safety Observation}

Safety assessment was based on parents' spontaneous reports of adverse events and vital signs.

\section{Sample Size Estimation}

The sample size we set to provide adequate power to detect differences of time to cough resolution between the two groups. According to the opinions of pediatric experts and the results of a previous study, the average time for PPACCM to relieve severe URTIassociated cough in children is $3.6 \pm 1.2$ days. ${ }^{(16)}$ On this basis, the difference of at least 0.5 day is accepted in the routine clinical practice of treating URTI-associated cough. Therefore, 63 participants per study group provided $80 \%$ power with a value of 0.05 to detect a significant difference of half a day or more in time to cough resolution. Assuming a 10\% dropout rate, a total of 140 participants were required.

\section{Statistical Analyses}

An intention-to-treat analysis was performed. All patients who were randomly assigned and received at least one treatment were included in the effectiveness and safety analyses. All outcomes were analyzed by a statistician blinded to treatment assignments. Mean \pm standard deviation $(\bar{x} \pm s)$ or medians and interquartile ranges (IQR) were calculated to summarize continuous variables, and the proportion of patients in each category was calculated to summarize categorical variables. Characteristics and baseline were compared among the two groups using the independent-samples $t$-test for continuous measures or the Wilcoxon-MannWhitney test for non-normal distributions and the Chisquare test for categorical variables.

For analyses of difference in primary outcomes, the Kaplan-Meier method with log-rank testing was used to describe and compare the median and interquartile range (IQR) of the time to cough resolution. Cox proportional hazards regression model for censored data was used to estimate the treatment effect, with cough resolution as the dependent variable and age, sex, body mass index, past medical history, exposure factors, total symptom score, and severity of cough as the independent variables. 
Hazard ratios (HRs) and corresponding 2-sided 95\% confidence intervals (Cls) were generated. Chi-square test was used to compare the 4-day cough resolution rate between groups, and relative risk (RR) and risk differences with $95 \% \mathrm{Cl}$ were calculated. In addition, changes in cough resolution rate from day 1 to 5 were assessed with a generalized linear mixed model with using PROC GLIMMIX in SAS (version 9.1.3, SAS Institute, Cary, North Carolina, USA). The interaction of time with treatment and center with treatment were also analyzed in the model. Group differences in the secondary outcomes were tested at day 1 to 5 using independent-samples t-test.

A $P$-value $<0.05$ was considered statistically significant. All analyses were performed by using IBM SPSS Statistics for Windows (version 20.0, USA), except for analyses of changes in cough resolution rate from day 1 to 5 , which were performed using SAS software.

\section{RESULTS}

\section{Patient Characteristics}

A total of 142 participants with moderate to severe URTI-cough aged 2 to 14 years old were randomly assigned to the JWZH group $(n=73)$ or PPACCM group $(n=69)$ between December 2019 and March 2021. After randomization, 3 children were identified as ineligible, 2 of whom were under 2 years old, and 1 had a cough score of less than 2; 1 eligible children in the JWZH group did not take the medication due to self-reasons. Therefore totally 138 participants were included in intention-to-treat population, $95.8 \%(68 / 71)$ of participants in the JWZH group and $94.0 \%(63 / 67)$ in the PPACCM group completed the study, while 7 withdrew from the trial due to unsatisfying effectiveness or adverse events (Figure 1). The baseline characteristics of the participants are shown in Table 1. There were no significant differences between the groups in demographic characteristics and baseline of disease $(P>0.05)$. The proportion of fever case and use of antipyretic were similar between the two groups $(15.5 \%$ to $11.9 \%, P=0.237 ; 14.1 \%$ to $11.9 \%$, $P=0.709$, Table 1).

\section{Primary Outcomes}

In the multivariate Cox model, children receiving JWZH recovered faster than those receiving PPACCM, after adjustment for age, sex, body mass index, severity, and previous medical history and

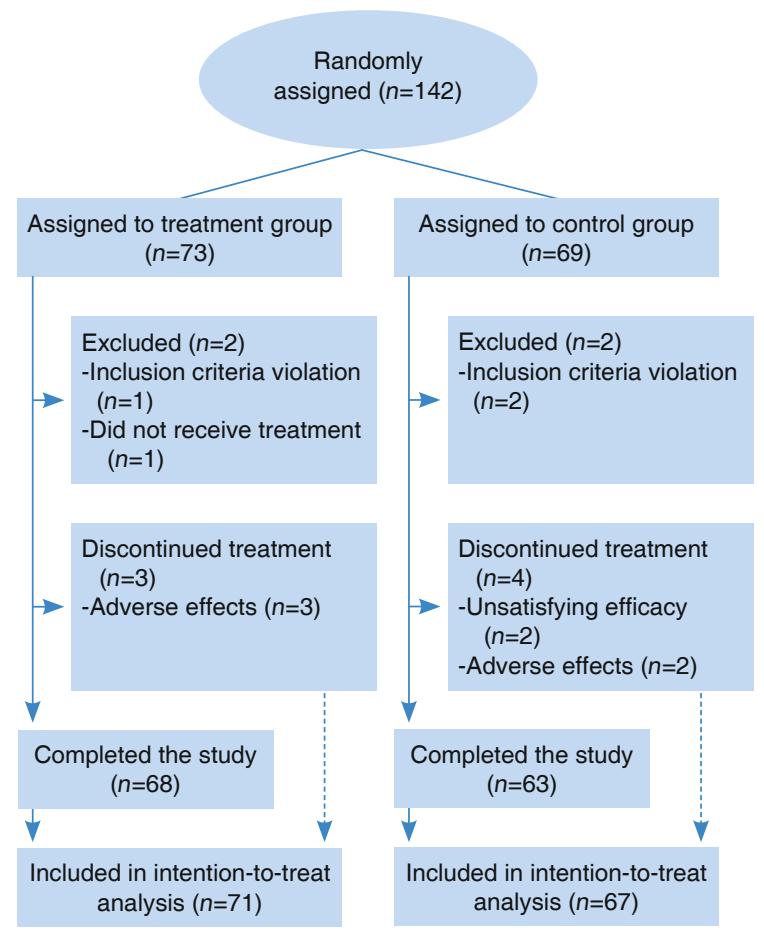

Figure 1. Enrollment, Randomization, and Follow-up in the Trial of JWZH for Children with URTI Notes: URTI: upper respiratory tract infections; the same below

stratification by center (HR, 2.10; $95 \% \mathrm{Cl}, 1.29-3.40$; $P=0.003$ ). A sub-group analysis based on cough severity was conducted and the results showed similar effect. The median time to cough resolution was 2.0 days $(95 \% \mathrm{Cl}, 1.9-2.1)$ for children receiving JWZH and 3.0 days $(95 \% \mathrm{Cl}, 2.7-3.3)$ for children receiving PPACCM. Therefore, the median time to cough resolution was 1 day, or $33.3 \%$ shorter in the children receiving JWZH (Table 2 and Figure 2).

The 4-day cough resolution rate was $94.4 \%$ $(67 / 71)$ in the JWZH group and $74.6 \%(50 / 67)$ in the PPACCM group. The former was $19.8 \%$ (95\% Cl: $8.1 \%-31.5 \%$ ) higher than the latter with a significant difference (RR: $1.265,95 \% \mathrm{Cl}: 1.088-1.470, P=0.001$, Table 3).

\section{Secondary Outcomes}

The scores of daytime cough from day 2 to 5 and nighttime cough from day 1 to 4 were statistically different between the two groups (all $P<0.01$ ). The scores of cough symptoms in the JWZH group were lower than those in the PPACCM group. There was no statistical difference in the scores of fever, nasal congestion, runny nose, and sneezing between the two groups from day 1 to 5 (all $P>0.05$ ). The detailed 
Table 1. Baseline Characteristics of Participants in Intention-to-Treat Analysis

\begin{tabular}{|c|c|c|}
\hline Characteristics & $\begin{array}{l}\text { JWZH } \\
\text { (71 cases) }\end{array}$ & $\begin{array}{l}\text { PPACCM } \\
\text { (67 cases) }\end{array}$ \\
\hline Age [Year, median (IQR)] & $3.8(2.6-5.7)$ & $4.5(3.3-6.8)$ \\
\hline 2-3 years [Case $(\%)]$ & 38 (53.5) & $27(39.7)$ \\
\hline 4-7 years [Case $(\%)]$ & $21(29.6)$ & $33(48.5)$ \\
\hline $8-14$ years [Case (\%)] & $12(16.9)$ & $8(11.8)$ \\
\hline \multicolumn{3}{|l|}{ Sex [Case (\%)] } \\
\hline Male & $41(57.7)$ & $33(49.3)$ \\
\hline Female & $30(42.3)$ & $34(50.7)$ \\
\hline Body mass index $\left[\mathrm{kg} / \mathrm{m}^{2}, \bar{x} \pm s\right]$ & $16.1 \pm 2.0$ & $15.8 \pm 1.9$ \\
\hline $\begin{array}{l}\text { Number of URTIs in the past year } \\
(\bar{x} \pm s)\end{array}$ & $2.5 \pm 1.1$ & $2.9 \pm 1.3$ \\
\hline $\begin{array}{l}\text { Hospitalization history of } \\
\text { respiratory diseases [Case (\%)] }\end{array}$ & $24(33.8)$ & $24(35.8)$ \\
\hline \multicolumn{3}{|l|}{ Underlining condition [Case (\%)] } \\
\hline Variant cough & $1(1.4)$ & $2(3.0)$ \\
\hline Asthma & $3(4.2)$ & $1(1.5)$ \\
\hline Tobacco smoke exposure & $29(40.8)$ & $35(52.2)$ \\
\hline $\begin{array}{l}\text { Total symptom score } \\
\text { [Median (IQR)] }\end{array}$ & $6(4-9)$ & $5(4-6)$ \\
\hline Total cough score* [Median (IQR)] & $4(3-5)$ & $4(3-5)$ \\
\hline \multicolumn{3}{|l|}{ Severity of cough [Case (\%)] } \\
\hline Moderate (Score: 2 to 4 ) & $43(60.6)$ & $44(65.7)$ \\
\hline Severe (Score: 5 to 6 ) & $28(39.4)$ & $23(34.3)$ \\
\hline \multicolumn{3}{|l|}{ Temperature [Case (\%)] } \\
\hline$<37.2{ }^{\circ} \mathrm{C}$ & $60(84.5)$ & $59(88.1)$ \\
\hline $37.2-38.5^{\circ} \mathrm{C}$ & $6(8.5)$ & $7(10.4)$ \\
\hline$>38.5^{\circ} \mathrm{C}$ & $5(7.0)$ & $1(1.5)$ \\
\hline Nasal congestion [Case (\%)] & $36(50.7)$ & $35(52.2)$ \\
\hline Runny nose [Case (\%)] & $40(56.3)$ & $41(59.7)$ \\
\hline Sneeze [Case (\%)] & $29(40.8)$ & $20(29.9)$ \\
\hline Use of antipyretic [Case (\%)] & $10(14.1)$ & $8(11.9)$ \\
\hline
\end{tabular}

Note: *Sum of daytime and nighttime cough scores

Table 2. Median Time to Cough Resolution in Children with URTI (d)

\begin{tabular}{|c|c|c|c|c|c|c|}
\hline \multirow[b]{2}{*}{ Group } & \multicolumn{2}{|r|}{ Total } & \multicolumn{2}{|c|}{ Moderate } & \multicolumn{2}{|c|}{ Severe } \\
\hline & Case & $\begin{array}{l}\text { Duration } \\
(95 \% \mathrm{Cl})\end{array}$ & Case & $\begin{array}{l}\text { Duration } \\
(95 \% \mathrm{Cl})\end{array}$ & Case & $\begin{array}{l}\text { Duration } \\
(95 \% \mathrm{Cl})\end{array}$ \\
\hline JWZH & 71 & $\begin{array}{c}2.0 \\
(1.9-2.1)\end{array}$ & 43 & $\begin{array}{c}2.0 \\
(1.7-2.3)\end{array}$ & 28 & $\begin{array}{c}2.0 \\
(1.5-2.5)\end{array}$ \\
\hline PPACCM & 67 & $\begin{array}{c}3.0 \\
(2.7-3.3)\end{array}$ & 28 & $\begin{array}{c}3.0 \\
(2.4-3.6)\end{array}$ & 23 & $\begin{array}{c}4.0 \\
(3.5-4.5)\end{array}$ \\
\hline$P$ value & & $<0.001$ & & $<0.001$ & & 0.003 \\
\hline
\end{tabular}

changes in symptom scores of URTI from day 1 to 5 are shown in Appendix 3.

In the generalized linear mixed model, changes in

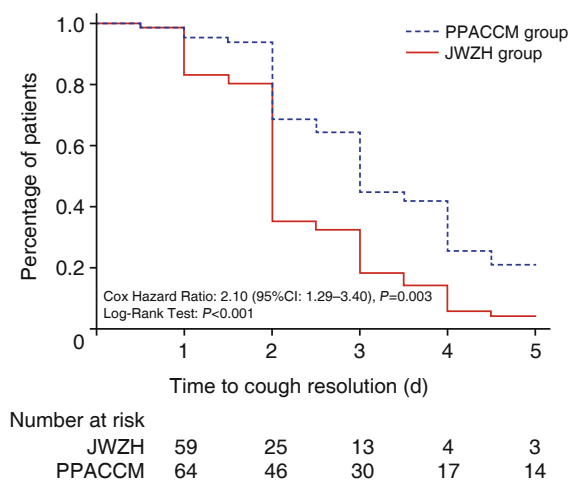

Figure 2. Kaplan-Meier Analysis of Effect of JWZH on Time to Cough Resolution in Children

Table 3. Changes of Cough Resolution Rate in Children with URTI from Day 1 to 5 [Case (\%)]

\begin{tabular}{|c|c|c|c|c|c|}
\hline $\begin{array}{l}\text { Time } \\
\text { point }\end{array}$ & $\begin{array}{c}\text { JWZH } \\
\text { (71 cases) }\end{array}$ & $\begin{array}{l}\text { PPACCM } \\
\text { (67 cases) }\end{array}$ & $\begin{array}{c}\text { Difference } \\
(\%, 95 \% \mathrm{Cl})\end{array}$ & RR (95\% Cl) & $P$ value \\
\hline Day 1 & 12 (16.9) & $3(4.5)$ & $\begin{array}{c}12.4 \\
(2.4-22.4)\end{array}$ & $\begin{array}{c}3.775 \\
(1.114-12.789)\end{array}$ & 0.019 \\
\hline Day 2 & $46(64.8)$ & 21 (31.3) & $\begin{array}{c}33.5 \\
(17.8-49.2)\end{array}$ & $\begin{array}{c}2.067 \\
(1.394-3.064)\end{array}$ & $<0.001$ \\
\hline Day 3 & $58(81.7)$ & 37 (55.2) & $\begin{array}{c}26.5 \\
(11.6-41.4)\end{array}$ & $\begin{array}{c}1.479 \\
(1.161-1.884)\end{array}$ & $<0.001$ \\
\hline Day 4 & 67 (94.4) & $50(74.6)$ & $\begin{array}{c}19.8 \\
(8.1-31.5)\end{array}$ & $\begin{array}{c}1.265 \\
(1.088-1.470)\end{array}$ & 0.001 \\
\hline Day 5 & 68 (95.8) & $53(79.1)$ & $\begin{array}{c}16.7 \\
(5.9-27.5)\end{array}$ & $\begin{array}{c}1.211 \\
(1.061-1.382)\end{array}$ & 0.003 \\
\hline
\end{tabular}

cough resolution rate from day 1 to 5 were statistically significant $(P=0.0074)$. On day 5 , the last day of the treatment, the cough resolution rate of the JWZH group was $95.8 \%$, which was higher than $79.1 \%$ of the PPACCM group $(P=0.003)$. The interaction effects between treatment and center and between treatment and time were not statistically significant $(P=0.8864$, $P=0.6697$, respectively, Table 3 and Figure 3).

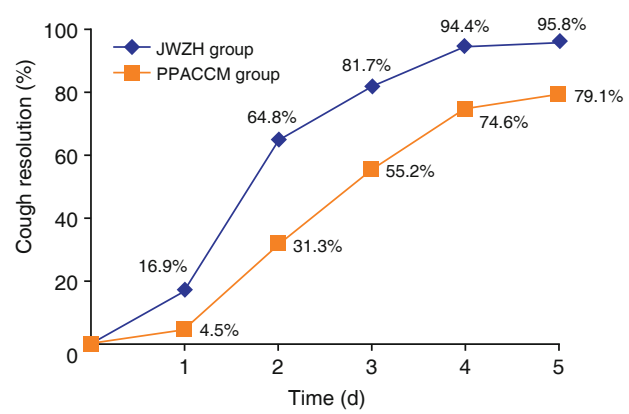

Figure 3. Changes of Cough Resolution Rate in Children with URTI from Day 0 to 5

\section{Safety Observation}

Adverse events were reported in 5.6\% (4/71) participants in the JWZH group and $4.5 \%(3 / 67)$ in the PPACCM group. Two patients in each group withdrew from the trial due to adverse events. All 
adverse events were mild and resolved without treatment. No serious events were found in both groups (Table 4).

Table 4. Summary of Adverse Events [Case (\%)]

\begin{tabular}{lccr}
\hline Adverse events & $\begin{array}{c}\text { JWZH } \\
(71 \text { cases })\end{array}$ & $\begin{array}{c}\text { PPACCM } \\
\text { (67 cases) }\end{array}$ & $P$ value \\
\hline Vomiting & $1(1.4)$ & $1(1.5)$ & $>0.999$ \\
Diarrhea & $1(1.4)$ & $1(1.5)$ & $>0.999$ \\
Increased stool frequency & $3(4.2)$ & $1(1.5)$ & 0.654 \\
Fever & 0 & $1(1.5)$ & 0.486 \\
\hline
\end{tabular}

\section{DISCUSSION}

There are few registered RCT in the field of pediatrics of Tibetan medicine. The results of our RCT demonstrated that the application of JWZH was associated with the shortening of cough duration and the improvement of cough resolution rate in children with URTI-associated cough. Currently, a series of reviews of the most recent evidence showed that the best therapeutic approaches for children and adolescents with acute cough remain unclear, ${ }^{(5,17,18)}$ and the natural duration of URTI-associated cough in children often continues for 1-2 weeks or even more. ${ }^{(3,19)}$ Acute cough and other associated symptoms of UTRI can be safely managed by using non-sedative medications especially natural compounds including honey, ${ }^{(5)}$ glycerol, and herb-derived components. ${ }^{(17)}$ Therefore, this finding may have a positive clinical significance for the management of cough in children with URTI.

The effect of JWZH for treating cough in our study was consistent with and slightly superior to a previous case series study conducted in the Tibet, ${ }^{(11)}$ in which the total cough score decreased from 3.17 to 0.74 after 5 days of treatment. The difference in effectiveness between the two studies might due to the different conditions of participant, since $61.7 \%(37 / 60)$ of children included in the case series study were complicated with acute bronchitis or pneumonia. Besides, the study found that JWZH had similar effects with PPACCM on the symptoms of nasal congestion, runny nose, and sneezing in children with URTI, which was similar with another case series study evaluating the effect of JWZH in treating 50 cases of children with common cold. ${ }^{(12)}$

JWZH is a classic formula commonly used by Tibetan medicine for hundreds of years to treat coughs caused by respiratory tract infections in children.
It is a well-known medication composed of natural medicinal herbs. The possible mechanism underlying the beneficial effects of JWZH may mediated via synergistic regulation of several biological pathways, such as signal transduction, immune system, cell growth, apoptosis, cancer, and other related signaling pathways. ${ }^{(20)}$ A pharmacodynamics study showed that JWZH power had a significant inhibitory effect on influenza A virus Tianjin Jinnan/15/2009 strain $\left(\mathrm{H}_{1} \mathrm{~N}_{1}\right.$ subtype $)$ and CoxB3, and slight inhibitory effect on HSV-1 and rhinovirus in vitro; its mechanism might involve the reduction of neuraminidase activity (Xie JR, personal communication, 2021).

In this study, since the current guidelines did not recommend specific drugs for children with URTI-associated cough, and placebo would not be accepted by Chinese parents, we used PPACCM as control, which was one of the most commonly used over-the-counter medications and recommended by the guidelines for URTI in children in China. ${ }^{(14)}$ It is composed of paracetamol, atificial cow-bezoar and chlorphenamine maleate, with antipyretic, analgesic and antihistamine effects. The results of this study showed that the median cough resolution time of PPACCM for children with moderate to severe URTIassociated cough was 3 days, which was consistent with the average of $3.6 \pm 1.2$ days reported in a previous study. ${ }^{(16)}$

Our study has some limitations. First, it was not a double blind placebo-controlled trial. JWZH was given as powder, and PPACCM was given as granules. Since PPACCM is a commonly used over-the-counter medication in pediatrics, it can be easily distinguished by Chinese children and their parents, and JWZH, a Tibetan medicinal patent medicine, has a unique smell, we could not find appropriate placebos for JWZH and PPACCM that had a similar form, color and taste. So we used an open-label, positive drug control design. Although it cannot be completely ruled out that the placebo effect may cause a shorter duration of cough, it still has application value for evaluating the effectiveness of JWZH in the real world. Second, there was a potential selective bias of participants. The study began to recruit participants in December 2019; however, after the outbreak of COVID-19 in January 2020, all patients with fever were placed in fever clinics according to China's epidemic prevention and control policy, then only children with URTI who 
did not have fever at their first visit, were available for inclusion in this study. Third, outcome measurement was subjective and based on the self-reporting of parents. To avoid potential biases of self-reporting, we used cough resolution as the endpoint indicator, and it was defined as the cough disappeared continuously for $\geqslant 24 \mathrm{~h}$. Therefore, the use of the two primary outcomes, although both subjective, may increase the credibility and repeatability of this trial. Besides, the research assistants who contacted the parents of participants were unaware of study group assignment.

In conclusion, in this trial, JWZH appeared to be a safe and effective therapy for decreasing the time to cough resolution and improving the 4-day cough resolution rate in children over 2 years old with URTI-associated cough. However, the absence of a placebo control weakened the evidence for effectiveness assessment of JWZH. A multicenter double-blind double-dummy RCT is still warranted to further confirm its effectiveness.

\section{Conflict of interest}

The authors declare that they have no competing interest.

\section{Author contributions}

Luo H, Peng S, and Song GH conceptualized and designed the study. Song GH, Ma XJ, Sun MM, Zhang M, and Peng $S$ undertook the trial. Luo $\mathrm{H}$ and $\mathrm{Xie} J \mathrm{~J}$ involved in the analysis and interpretation of the data. Luo $\mathrm{H}$ drafted the initial manuscript. All authors read and approved the final manuscript. Luo $\mathrm{H}$ and Song $\mathrm{GH}$ contributed equally to this work.

Electronic Supplementary Material: Supplementary material (Appendices 1-3) is available in the online version of this article at https://doi.org/10.1007/s11655-021-3462-x.

\section{REFERENCES}

1. Cotton $\mathbf{M}$, Innes $\mathrm{S}$, Jaspan $\mathrm{H}$, Madide A, Rabie H. Management of upper respiratory tract infections in children. S Afr Fam Pract (2004) 2008;50:6-12.

2. Pappas DE, Hendley JO, Hayden FG, Winther B. Symptom profile of common colds in school-aged children. Pediatr Infect Dis J 2008;27:8-11.

3. Hay $A D$, Wilson AD. The natural history of acute cough in children aged 0 to 4 years in primary care: a systematic review. Br J Gen Pract 2002;52:401-409.

4. Murgia V, Manti S, Licari A, De Filippo M, Ciprandi G, Marseglia GL. Upper respiratory tract infection-associated acute cough and the urge to cough: new insights for clinical practice. Pediatr Allergy Immunol Pulmonol 2020;33:3-11.

5. Abuelgasim H, Albury C, Lee J. Effectiveness of honey for symptomatic relief in upper respiratory tract infections: a systematic review and meta-analysis. BMJ Evid Based Med 2021;26:57-64.

6. Paul IM, Beiler J, McMonagle A, Shaffer ML, Duda L, Berlin CJ. Effect of honey, dextromethorphan, and no treatment on nocturnal cough and sleep quality for coughing children and their parents. Arch Pediatr Adolesc Med 2007;161:1140-1146.

7. Morice A, Kardos P. Comprehensive evidence-based review on European antitussives. BMJ Open Respir Res 2016;3:e137.

8. Smith SM, Schroeder K, Fahey T. Over-the-counter (OTC) medications for acute cough in children and adults in community settings. Cochrane Database Syst Rev 2014;2014:D1831.

9. Fashner J, Ericson K, Werner S. Treatment of the common cold in children and adults. Am Fam Physician 2012;86:153-159.

10. Luo H. Traditional Tibetan medicine in China: an overview of current status and prospects. Chin J Integr Med 2015;21:67-70.

11. Da Z. Tibetan medicine "Jiu Wei Zhu Huang San" in the treatment of 60 children with acute respiratory Infection. World Latest Med Inf (Electr, Chin) 2018;98:290-292.

12. Suonan AX. Tibetan medicine Jiuwei Zhuhuang Granules in treating 50 children with common cold and fever. J Med Pharm Chin Minor (Chin) 2014;20(5):12.

13. Lai KF. The cough diagnosis and treatment guidelines (2015). Chin J Tubercul Respir Dis (Chin) 2016;39:323-354.

14. An SH, Ai T, Bao YX, Chen DH, Chen HZ, Chen Q, et al. Expert consensus on standardized diagnosis and treatment of common cold in children in China (2013). Chin J Pract Pediatr (Chin) 2013;28:680-686.

15. Ma R, Hu S, Ding Y, Xiang X, Yang J, Xue Z, et al. The design and evaluation technology of new Chinese medicines for acute upper respiratory infections in children. Drug Evaluat Res (Chin) 2015;38:8-16.

16. Tao LH, Guan MC, Peng CJ, Jin $\mathrm{XH}$. The therapeutic efficacy of pediatric paracetamol combined with ribavirin aerosol in children with acute upper respiratory tract infection. China Med (Chin) 2015;10:177-179.

17. Marseglia GL, Manti S, Chiappini E, Brambilla I, Caffarelli C, Calvani $\mathrm{M}$, et al. Acute cough in children and adolescents: a systematic review and a practical algorithm by the Italian Society of Pediatric Allergy and Immunology. Allergol Immunopathol (Madr) 2021;49:155-169.

18. Manti S, Tosca MA, Licari A, Brambilla I, Foiadelli T, Ciprandi $G$, et al. Cough remedies for children and adolescents: current and future perspectives. Paediatr Drugs 2020;22:617-634.

19. Jones BF, Stewart MA. Duration of cough in acute upper respiratory tract infections. Aust Fam Physician 2002;31:971-973.

20. Guo SY, Zhou W, Wu JR, Liu XK, Meng ZQ, Tian JH, et al. Network pharmacology-based study on the mechanism of "Jiu Wei Zhu Huang San" in respiratory tract infections treatment. Eur J Integr Med 2019;32:101013.

(Accepted November 9, 2021; First Online December 15, 2021) Edited by YUAN Lin 